\title{
EDITORIAL
}

\section{Global effort against rare and orphan diseases}

\author{
Sergio Harari*, Vincent Cottin ${ }^{\#}$ and Marc Humbert
}

$\mathbf{T}$ he fifth International Congress on Rare Pulmonary Diseases and Orphan Drugs will take place in Milan, Italy, on February 8-9, 2013 (www.pulmonaryrarediseases. com). 10 yrs have passed since the first Congress was held. Since then, the Congress has taken place regularly every 2 yrs, with growing success and ever increasing numbers of participants and speakers from around the world. The International Congress on Rare Pulmonary Diseases and Orphan Drugs is the only European event dedicated to different types of rare pulmonary diseases affecting both parenchymal and vascular structures. Overall, more than 150 speakers and 650 participants have attended the past four Congresses, where scientific programmes are designed to build bridges between physicians specialised in the management of different lung disorders that have the common feature of being rare diseases, i.e. with prevalence lower than one per 2,000 persons according to the European definition.

Proceedings from the 2005 and 2009 Congresses have been published previously elsewhere [1, 2]. In the present issue of the European Respiratory Review (ERR), three selected articles focus on some of the most important and widely discussed topics from the fourth Congress held on February 25-26, 2011 [3-5]. These manuscripts summarise the two main scientific areas of discussion: 1) the basic science research, and 2) the prognostic evaluation and management of pulmonary vascular and interstitial lung diseases. In addition, several other important topics were discussed during the 2-day Congress, including original scientific data and results of clinical trials with new drugs that provided evidence for innovative therapeutic approaches to rare pulmonary diseases. New developments in the treatment of pulmonary arterial hypertension $(\mathrm{PAH})$, and critical issues on idiopathic pulmonary fibrosis (IPF), sarcoidosis, rheumatoid arthritis, lung involvement in systemic sclerosis, pulmonary Langerhans' cells histiocytosis and lymphangioleiomyomatosis (LAM) were extensively discussed by outstanding international speakers.

The three papers published in this issue of the ERR illustrate the different clinical and translational issues that were presented

\footnotetext{
*Unità Operativa di Pneumologia e Terapia Semi-Intensiva Respiratoria -Servizio di Fisiopatologia Respiratoria ed Emodinamica Polmonare-Ospedale San Giuseppe MultiMedica, Milan, Italy. ${ }^{\#}$ Centre National de référence des maladies pulmonaires rares et Centre de compétences de l'hypertension artérielle pulmonaire, Hospices Civils de Lyon, Service de pneumologie, Hôpital Louis Pradel, Université de Lyon, Université Claude Bernard Lyon I, INRA, UMR754 IFR 128, Lyon, and "Centre National de Réérence de l'Hypertension Pulmonaire Sévère, Hôpital Bicêtre, Hôpitaux Universitaires Paris-Sud, Assistance Publique Hôpitaux de Paris, Le Kremlin-Bicêtre, France.
}

CORRESPONDENCE: S. Harari, Unità Operativa di Pneumologia e Terapia Semi-Intensiva Respiratoria -Servizio di Fisiopatologia Respiratoria ed Emodinamica Polmonare-Ospedale San Giuseppe MultiMedica, Via San Vittore 12, 20123 Milan, Italy. E-mail: sharari@hotmail.it

PROVENANCE: Submitted article, peer reviewed. and discussed at the Congress. CAMINATI et al. [3] propose and discuss a multidisciplinary and integrated approach to smokingrelated interstitial lung diseases, O'CALLAGHAN and HUMBERT [4] delineate the critical issues in the evaluation of survival in patients with PAH and, finally, GLASGOW et al. [5] review the multiple aspects of lymphatic involvement of LAM and IPF.

In recent years, the interest for rare diseases has grown throughout the entire medical community, and many respiratory physicians were pioneers in this field of medicine. Data clearly demonstrate this emerging awareness: in 1989-1999 a total of 114 patients were enrolled in four well-conducted IPF studies, whereas in the following decade (2000-2010) almost 3,000 patients were enrolled in a total of 11 studies [6]. A search of articles written in English in PubMed with the keywords "idiopathic pulmonary fibrosis" "lymphangioleiomyomatosis" and "pulmonary Largerhans' cells histiocytosis" shows a growing numbers of papers published in recent years (table 1), while the search for rare pulmonary disease studies in the Clinicaltrials.gov website retrieved a total of 114 trials on IPF and 18 on LAM. If we consider the rarity of these diseases and the state-ofthe-art research just a few years ago, these data represent a substantial (and astonishing) number of clinical studies.

However, by far the paradigm for success of the research in this field and in the development of orphan drugs is PAH. This disease has greatly benefited from the establishment of an international network of research focused on understanding the complex pathogenesis and development of the disease. The registration of eight different drugs, with important impact on the prognosis as well as the quality of life of patients suffering from this disease, demonstrates the strong effort made in the last 20 yrs to help clinicians, patients and relatives fight this devastating pulmonary vascular disease.

The tremendous progress made in the field of rare pulmonary diseases has been made possible by a number of factors including, among others: 1) better delineation of some of these diseases (especially IPF); 2) the collective research effort by the respiratory community; 3 ) the clinical research effort of collaborative groups, such as the Groupe d'Etudes et de Recherche sur les Maladies "Orphelines" Pulmonaires [7]; 4) the tremendous effort of the public, physicians and patients, and the progress in the terminology of rare diseases by Orphanet (www.orpha.net); 5) the emergence of coordinated action against rare diseases by health authorities in several western countries and at European level; 6) the role of patient associations, which have raised awareness, money and, in some cases, facilitated clinical trials [8]; and 7) the investment by major pharmaceutical companies, themselves fostered by orphan drug legislation. 


\begin{tabular}{|c|c|c|c|c|}
\hline Keywords & $1980-1989$ & 1990-1999 & 2000-2009 & 2010-May 2012 \\
\hline Idiopathic pulmonary fibrosis & 249 & 577 & 1577 & 770 \\
\hline Lymphangioleiomyomatosis & 39 & 170 & 472 & 163 \\
\hline Pulmonary Langerhans' cell histiocytosis & 142 & 201 & 291 & 74 \\
\hline
\end{tabular}

During the 2011 European Respiratory Society Congress in Amsterdam, the Clinical Assembly of the society organised a symposium dedicated to these topics, the proceeding of which were published in the European Respiratory Journal [9]. The increasing interest of the ERR in this field is also reflected by the 23 articles published on IPF since 2005, and the 31 articles on PAH and three on LAM published in the last 2 yrs.

The decision of the ERR to publish these reports from the 4th International Congress on Rare Pulmonary Diseases and Orphan Drugs goes further in this direction, highlighting that rare diseases represent an important field of medicine not only for pulmonologists who are skilled in diagnosing and treating particular groups of these illnesses, but for all respiratory physicians. Knowledge exchange and dissemination among experts on different areas of clinical and basic research in respiratory medicine, with different specialised pulmonary medicine expertise (from vascular to interstitial lung diseases), is part of the stimulating challenge of providing new insights into science and clinical care, thus helping patients and supporting doctors.

\section{STATEMENT OF INTEREST}

S. Harari has relationships with drug companies including Actelion, InterMune and GSK. In addition to being investigator in trials involving these companies, relationships include consultancy service and membership of scientific advisory boards. V. Cottin has received fees for speaking from Actelion, Boeringer Ingelheim, GSK and Pfizer, and has participated as investigator to clinical trials sponsored by Actelion, Boeringer Ingelheim, GSK and Pfizer. He has served on advisory boards for Actelion, Bayer, Boeringer Ingelheim, InterMune, Lilly and Novartis. M. Humbert has relationships with drug companies including Actelion,
Aires, AstraZeneca, Bayer, Bristol Myers Squibb, GSK, Merck, Novartis, Nycomed, Pfizer, Stallergènes, TEVA and United Therapeutics. In addition to being investigator in trials involving these companies, relationships include consultancy service and membership of scientific advisory boards.

\section{REFERENCES}

1 Harari S, Agostini C. New frontiers for rare pulmonary diseases. Sarcoidosis Vasc Diffuse Lung Dis 2005; 22: Suppl. 1, S1-S3.

2 Harari S, Agostini C. Proceedings of the 3rd International Congress on Rare Pulmonary Diseases and Orphan Drugs Milan, Italy. Respir Med 2010; 104: Suppl. 1, S1-S92.

3 Caminati A, Cavazza A, Sverzellati N, et al. An integrated approach in diagnosis of smoking-related interstitial lung diseases. Eur Respir Rev 2012; 21: 207-217.

4 O'Callaghan DS, Humbert M. A critical analysis of survival in pulmonary arterial hypertension. Eur Respir Rev 2012; 21: 218-222.

5 Glasgow CG, El-Chemaly S, Moss J. Lymphatics in lymphangioleiomyomatosis and idiopathic pulmonary fibrosis. Eur Respir Rev 2012; 21: 196-206.

6 Cottin V. Changing the idiopathic pulmonary fibrosis treatment approach and improving patient outcomes. Eur Respir Rev 2012; 21: 161-167.

7 du Bois RM. Rare lung diseases: orphan no more? Am J Respir Crit Care Med 2002; 156: 1557-1558.

8 Ingelfinger JR, Drazen JM. Patient organizations and research on rare diseases. N Engl J Med 2011; 364: 1670-1671.

9 Spruit MA, Chavannes NH, Herth FJF, et al. Clinical highlights from the 2011 ERS Congress in Amsterdam. Eur Respir J 2012; 39: 1501-1510. 